\title{
BATF2 and FOXJ1 are differentially expressed in coronavirus infections.
}

Shahan Mamoor ${ }^{1}$

'shahanmamoor@gmail.com

East Islip, NY USA

Unraveling the host transcriptional response to viral infections is important for understanding host-pathogen interactions. We mined published microarray datasets ${ }^{1-5}$ to identify conserved and specific differentially expressed genes in in vitro and in vivo models of coronavirus infections. We found significant transcriptional induction of the transcription factors BATF2 and FOXJ1 in Middle East Respiratory Syndrome (MERS) coronavirus infection in human cells in vitro; BATF2 was also differentially expressed in the lungs of mice infected with the Severe Acute Respiratory Syndrome (SARS) coronavirus 1 (SARS-CoV-1) but not in human cells infected with the human coronavirus HCoV-229E. These data highlight specific host induction of transcription factors by different members of the coronavirus family.

Keyword: SARS-CoV-1, SARS-CoV-2, COVID-19, MERS-CoV, HCOV-229E, human coronavirus, coronavirus, systems biology of viral infection, targeted therapeutics in coronavirus infection. 
Nearly 200,000 deaths have resulted from infection with the novel coronavirus SARS-CoV-2 within one year in the United States ${ }^{6,7}$. Understanding the host transcriptional response to viral infection is critical for identification of novel therapeutic targets $^{8}$. We mined published microarray data ${ }^{1-5}$ to identify differentially expressed genes across a multitude of coronavirus infection models and found significantly transcriptional induction of the transcription factors BATF2 and FOXJ1 in human cells infected with the Middle East Respiratory Syndrome coronavirus MERS-CoV.

Differential expression of these transcription factors was not seen in human cells infected with human coronavirus HCoV-229E and was significantly less marked in the lungs of mice infected with the Severe Acute Respiratory Syndrome coronavirus SARS-

CoV-1, suggesting unique induction of transcription factor expression by host cells during coronavirus defense or by the coronavirus family.

\section{Methods}

We used datasets GSE56677', GSE59185², GSE68820³, GSE225814 and GSE891675 for this analysis of gene expression changes following coronavirus infection. GSE56677 was generated using Agilent-039494 SurePrint G3 Human GE v2 8x60K Microarray 039381 technology with $n=3$ each for CALU3 2B4 cells infected with MERS-CoV London (Eng1): at 0 hours, 3 hours, 7 hours, 12 hours, 18 hours and 24 hours post-infection. GSE59185 was generated using Agilent-028005 SurePrint G3 Mouse GE 8x60K Microarray technology with $n=3$ lung tissue from mock- 
infected BALB/c mice and $n=3$ lung tissue from SARS-CoV-1-infected BALB/c mice, both at 2 days post-infection. GSE68820 was generated using Agilent-014868 Whole Mouse Genome Microarray 4x44K G4122F technology with $n=5$ for lung tissue from mock-infected C57BL6/NJ mice and $n=5$ for lung tissue from SARS-CoV-1-infected C57BL6/NJ mice, both at 2 days post-infection. GSE22581 was generated using Affymetrix Canine Genome 2.0 Array technology with $n=3$ each for ferret lung on day 0 , day 1 and day 2 post-infection through the intranasal route with SARS-CoV-1. GSE89167 was generated using 039494 SurePrint G3 Human GE v2 8x60K Microarray 039381 technology with $n=2 \mathrm{HuH}-7$ cells and $n=2 \mathrm{HCoV}-229 \mathrm{E}$ infected $\mathrm{HuH}-7$ cells. The Benjamini and Hochberg method of $p$-value adjustment was used for ranking of differential expression but raw $p$-values were used for assessment of statistical significance of global differential expression. Log-transformation of data was autodetected, and the NCBI generated category of platform annotation was used.

A statistical test was performed to evaluate the significance of difference in FOXJ1 and BATF2 mRNA expression levels in CALU3 2B4 cells with MERS-CoV infection at 3 hours, 7 hours, 12 hours, 18 hours and 24 hours post-infection as compared to CALU3 2B4 cells at baseline (0 hours) using a one-way ANOVA with Dunnett's multiple comparisons test. Only $p$-values less than 0.05 were considered statistically significant. We used PRISM for all statistical analyses (Version 8.4.0)(455).

\section{Results}

We mined published microarray data ${ }^{1-5}$ to determine in an unbiased and 
systematic fashion the genes most differentially expressed in a series of human coronavirus infection models: in human cells infected with MERS-CoV or with human coronavirus 229E, and in the lungs of mice and ferrets infected with SARS-CoV-1.

BATF2 is differentially expressed in a human cell line following infection with an isolate of MERS-CoV, MERS-CoV London (Eng1).

We found that the basic leucine zipper transcription factor, ATF-like 2 (BATF2) was among the genes most differentially expressed following MERS-CoV London infection of the human cell line CALU3 2B4. When sorting each of the genes expressed in CALU3 cells based on change in expression between uninfected and MERS-CoVinfected cells, BATF2 ranked 102 out of 28653 total transcripts. Differential expression of BATF2 was statistically significant (Table $1 ; p=1.49 \mathrm{E}-12$ ).

FOXJ1 is differentially expressed in a human cell line following infection with an isolate of MERS-CoV, MERS-CoV London (Eng1).

We found a second transcription factor, forkhead box J1 (FOXJ1), among the genes most differentially expressed following MERS-CoV London infection of the human cell line CALU3 2B4. When sorting each of the genes expressed in CALU3 cells based on change in expression between uninfected and MERS-CoV-infected cells, FOXJ1 ranked 194 out of 28653 total transcripts. Differential expression of FOXJ1 was statistically significant (Table $1 ; p=9.27 \mathrm{E}-12$ ).

Infection of BALB/c mice with SARS-CoV-1 results in differential expression of BATF2, and to a lesser extent, FOXJ1 in the lungs.

BATF2 was also differentially expressed in the lungs of BALB/c mice infected 
with SARS-CoV-1, at 2 days post-infection. When sorting each of the genes expressed in the mouse lung based on change in expression between mock-infected and SARSCoV-1 mice, BATF2 ranked 118 out of 62976 total transcripts. Differential expression of BATF2 in the lungs of BALB/c mice following SARS-CoV-1 infection was statistically significant (Table 2; $p=3.06 \mathrm{E}-08$ ).

In the lungs of BALB/c mice infected with SARS-CoV-1, at 2 days post-infection, FOXJ1 was also differentially expressed, but markedly less significantly so than BATF2 was. When sorting each of the genes expressed in the mouse lung based on change in expression between mock-infected and SARS-CoV-1 mice, FOXJ1 ranked 14315 out of 62976 total transcripts. Differential expression of FOXJ1 in the lungs of BALB/c mice following SARS-CoV-1 infection was statistically significant (Table $2 ; p=4.92 \mathrm{E}-02$ ).

Infection of C57BL6/NJ mice with SARS-CoV-1 results differential expression of BATF2, and to a lesser extent, FOXJ1, in the lungs.

BATF2 was also differentially expressed in the lungs of C57BL6/NJ mice infected with SARS-CoV-1 at 2 days post-infection. When sorting each of the genes expressed in the mouse lung based on change in expression between mock-infected and SARSCoV-1 mice, BATF2 ranked 477 out of 29649 total transcripts. Differential expression of BATF2 in the lungs of C57BL6/NJ mice following SARS-CoV-1 infection was statistically significant (Table 3; $p=3.95 \mathrm{E}-10$ ).

In the lungs of C57BL6/NJ mice infected with SARS-CoV-1, at 2 days postinfection, FOXJ1 was differentially expressed, but again, markedly less significantly so 
than in human cells following infection with MERS-CoV London. When sorting each of the genes expressed in the mouse lung based on change in expression between mockinfected and SARS-CoV-1 mice, FOXJ1 ranked 17778 out of 29649 total transcripts. Differential expression of FOXJ1 in the lungs of C57BL6/NJ mice following SARS-CoV-1 infection was statistically significant (Table $3 ; p=1.68 \mathrm{E}-02$ ).

Infection of ferrets with SARS-CoV-1 does not result in differential expression of FOXJ1 but does result in statistically significant differential expression of BATF2 in the lungs.

BATF2 was also differentially expressed in the lungs of ferrets infected intranasally with SARS-CoV-1 at 2 days post-infection, but markedly less so than in mice. When sorting each of the genes expressed in the ferret lung based on change in expression between mock-infected and SARS-CoV-1 ferrets, BATF2 ranked 13353 out of 43045 total transcripts. Differential expression of BATF2 in the lungs of ferrets following SARS-CoV-1 infection was statistically significant (Table 4; $p=1.41 \mathrm{E}-02$ ).

In the lungs of ferrets infected intranasally with SARS-CoV-1, at 2 days postinfection, FOXJ1 was not differentially expressed. When sorting each of the genes expressed in the ferret lung based on change in expression between mock-infected and SARS-CoV-1 ferrets, FOXJ1 ranked 27340 out of 43035 total transcripts. Expression of FOXJ1 in the lungs of ferrets following SARS-CoV-1 infection was not significantly differential (Table 4; $p=1.7 \mathrm{E}-01$ ).

BATF2 and FOXJ1 are not differentially expressed in the blood of ferrets following intranasal SARS-CoV-1 infection. 
In the blood of ferrets infected intranasally with SARS-CoV-1, at 2 days postinfection, BATF2 was not differentially expressed. When sorting each of the genes expressed in the ferret blood based on change in expression between mock-infected and SARS-CoV-1 ferrets, BATF2 ranked 20307 out of 43035 total transcripts. Expression of BATF2 in the blood of ferrets following SARS-CoV-1 infection was not significantly differential (Table $5 ; p=4.06 \mathrm{E}-01$ ).

In the blood of ferrets infected intranasally with SARS-CoV-1, at 2 days postinfection, FOXJ1 was also not differentially expressed. When sorting each of the genes expressed in the ferret blood based on change in expression between mock-infected and SARS-CoV-1 ferrets, FOXJ1 ranked 14543 out of 43035 total transcripts. Expression of FOXJ1 in the blood of ferrets following SARS-CoV-1 infection was not significantly differential (Table $5 ; p=2.79 \mathrm{E}-01$ ).

Infection of a human cell line with $\mathrm{HCoV}-229 \mathrm{E}$ does not result in differential expression of FOXJ1 and BATF2.

Neither BATF2 nor FOXJ1 were significantly differentially expressed in the Huh-7 human cell line following infection with the human coronavirus 229E (HCoV-229E). BATF2 was the 8405 differentially expressed transcript out of 62976 transcripts total, and FOXJ1 was the 29221 differentially expressed transcript out of 62976 transcripts total. Neither BATF2 nor FOXJ1 was significantly differentially expressed (Table 6; $p=0.0991907$ and $p=0.398083$, respectively). 
Infection of a human cell line with an isolate of MERS-CoV results in rapid transcriptional induction of BATF2.

Next we assessed the kinetics of transcriptional changes in BATF2 following infection of CALU3 cells with MERS-CoV. At 3 hours and 7 hours, we observed decreases in BATF2 expression as compared to baseline; at 7 hours, this decrease approached the level of statistical significance (Figure $1 ; p=0.3494$ at 3 hours and $p=0.0557$ at 7 hours, respectively), with $0.9618 \pm 0.0091$ and $0.9364 \pm 0.0450$ foldchanges in BATF2 expression at 3 hours and 7 hours post-infection, respectively. At 12 hours post-infection, BATF2 expression increased and approached the level at which it was at baseline, with $0.9984 \pm 0.0310$ mean fold change in expression as compared to baseline (Figure 1; $p>0.9999$ ). However, from 12 hours to 18 hours, we observed a sharp increase in BATF2 expression, with a $1.4176 \pm 0.0173$ mean fold change in BATF2 expression as compared to baseline (Figure $1 ; p<0.0001$ at 18 hours). From 18 hours until 24 hours, BATF2 expression decreased somewhat but remained significantly increased as compared to baseline, with a $1.2624 \pm 0.0302$ mean fold change in BATF2 expression (Figure 1; $p=0.0001$ at 24 hours).

Infection of a human cell line with an isolate of MERS-CoV results in rapid transcriptional induction of FOXJ1.

We also assessed the kinetics of transcriptional changes in FOXJ1 following infection of CALU3 cells with MERS-CoV. From baseline ( 0 hours) to 12 hours, we did not detect much change in FOXJ1 expression; at 3 hours, the mean fold change in BATF2 expression was $1.0312 \pm 0.0231$, at 7 hours it was $1.0078 \pm 0.0180$, and at 12 
hours the mean fold change in BATF2 expression as compared to baseline was 1.0473 \pm 0.0299 (Figure 2; $p=0.3910$ at 3 hours and $p=0.9922$ at 7 hours, and $p=0.1071$ at 12 hours, respectively). At 18 hours post-infection, BATF2 expression increased and reach the level of statistical significance, with $1.0900 \pm 0.0249$ mean fold change in expression as compared to baseline (Figure 2; $p=0.0022$ ). From 18 to 24 hours, we observed a sharp increase in BATF2 expression, with a $1.4324 \pm 0.0265$ mean fold change in BATF2 expression as compared to baseline (Figure 2; $p<0.0001$ at 24 hours).

Infection of BALB/c mice with SARS-CoV-1 results in transcriptional induction of BATF2 in the lungs.

We also assessed transcriptional induction of BATF2 transcription factor mRNA in the lungs of BALB/c mice. BATF2 mRNA was present at significantly higher levels at 2 days post-infection with SARS-CoV-1, with a mean fold change of $1.3777 \pm 0.0299$ in BATF2 mRNA levels as compared to the lungs of mock-infected mice. Increased BATF2 mRNA in the lungs of BALB/c mice was statistically significant (Figure 3; $p=0.0016)$.

Thus we found that the transcription factors BATF2 and FOXJ1 were both among the genes most differentially expressed in a human cell line following infection with MERS-CoV London; BATF2 was also among the genes most differentially expressed in the lungs of two strains of mice infected with SARS-CoV-1. BATF2 and FOXJ1 were not significantly differentially expressed in the blood of ferrets following intranasal infection 
with SARS-CoV-1, nor were they differentially expressed in a human cell line following infection with the human coronavirus HCoV-229E. Within 18-24 hours of infection with MERS-CoV London, we observed significant transcriptional induction of both FOXJ1 and BATF2 in CALU3 cells. BATF2 was also transcriptionally induced in the lungs of BALB/c mice infected with SARS-CoV-1.

\section{Discussion}

Both of the transcription factors we identified here as differentially expressed following coronavirus infections have roles in the immune system; FOXJ1 also has established roles in airway epithelial cells.

CD4+ T-cells from FOXJ1-deficient mice produce significantly elevated amounts of the Th1 cytokines interleukin 2 (IL-2) and interferon- $\gamma(\text { IFN- } \gamma)^{9}$. NF-kB luciferase activity is significantly increased in primary T-cells from FOXJ1-deficient mice suggesting FOXJ1 functions as a regulator of NF-kB signaling in T-cells9. Lupus-prone MRL/lpr mice ectopically expressing FOXJ1 in the thymus using CD2-Cre possessed significantly decreased organ disease and autoantibody production, but this was not a result of NF-kB-modulating functions of FOXJ1 ${ }^{10}$. In mice, infection with Sendai virus, a virus closely related to the respiratory syncytial virus (RSV) results in significant reduction of the percentage of FOXJ1-positive airway epithelial cells as well as a reduction in FOXJ1 at the protein level11. Thus, viral infection can modulate FOXJ1 expression in the lungs, and FOXJ1 has roles in control of the Th1 cytokine response. 
M1-phenotype macrophages (activated by IFN- $\gamma$, or "classically" activated) express significantly higher levels of BATF2 as compared to unstimulated or M2phenotype macrophages (activated by IL-4) ${ }^{12}$. M1 macrophages depleted of BATF2 are compromised in their ability to produce mRNA for the inflammatory cytokine Tnf as well as the genes $\mathrm{Ccl} 5$ and Nos2 ${ }^{12}$. BATF2 mRNA is transcriptionally induced in macrophages infected with Mycobacterium tuberculosis ${ }^{12}$. The promoters of BATF2regulated genes are enriched in motifs for the interferon regulatory factor IRF1, and IRF1 and BATF2 can physically interact. BATF2 has also been described as a negative regulator of the cytokine interleukin 23a (IL-23a) ${ }^{13}$. CD4+ T-cells from the spleen and liver of BATF2-deficient mice infected with the parasite Trypanosoma cruzi produce significantly elevated amounts of IL-17 but not IFN- $\gamma$, and bone marrow-derived macrophages and dendritic cells from BATF2-deficient mice produced significantly elevated amounts of IL-23 ${ }^{13}$. Although parasite burden was decreased in T. cruziinfected BATF2-deficient mice, organ disease was significantly increased ${ }^{13}$. BATF2 could interact with c-JUN; this prevented c-JUN interaction and complex formation with ATF2, blocking IL23a transcriptional activation ${ }^{13}$. Thus, BATF2 is important for control of disease outcomes after pathogen infection in vivo and can control the production of cytokines from CD4+ T-cells and from innate immune cells like macrophages and dendritic cells.

We describe here the transcriptional induction of transcription factors BATF2 and FOXJ1 in human cells following infection with an isolate of MERS-CoV. BATF2 and 
FOXJ1 were also differentially expressed in the lungs of mice infected with SARS-

CoV1, but not in human cells following infection with HCoV-229E. BATF2 but not

FOXJ1 was not differentially expressed in the lungs of ferrets following infection with SARS-CoV-1. These data reveal unique induction of transcription factors important for the immune response following infection with different coronavirus family members. It remains unclear whether BATF2 and FOXJ1 are important for SARS-CoV-2 infection. 


\section{References}

1. Selinger, C., Tisoncik-Go, J., Menachery, V.D., Agnihothram, S., Law, G.L., Chang, J., Kelly, S.M., Sova, P., Baric, R.S. and Katze, M.G., 2014. Cytokine systems approach demonstrates differences in innate and pro-inflammatory host responses between genetically distinct MERS-CoV isolates. BMC genomics, 15(1), p.1161.

2. Regla-Nava, J.A., Nieto-Torres, J.L., Jimenez-Guardeño, J.M., Fernandez-Delgado, R., Fett, C., Castaño-Rodríguez, C., Perlman, S., Enjuanes, L. and DeDiego, M.L., 2015. Severe acute respiratory syndrome coronaviruses with mutations in the $E$ protein are attenuated and promising vaccine candidates. Journal of virology, 89(7), pp.3870-3887

3. Totura, A.L., Whitmore, A., Agnihothram, S., Schäfer, A., Katze, M.G., Heise, M.T. and Baric, R.S., 2015. Toll-like receptor 3 signaling via TRIF contributes to a protective innate immune response to severe acute respiratory syndrome coronavirus infection. MBio, 6(3), pp.e00638-15.

4. Danesh, A., Cameron, C.M., León, A.J., Ran, L., Xu, L., Fang, Y., Kelvin, A.A., Rowe, T., Chen, H., Guan, Y. and Jonsson, C.B., 2011. Early gene expression events in ferrets in response to SARS coronavirus infection versus direct interferon-alpha2b stimulation. Virology, 409(1), pp.102-112.

5. Poppe, M., Wittig, S., Jurida, L., Bartkuhn, M., Wilhelm, J., Müller, H., Beuerlein, K., Karl, N., Bhuju, S., Ziebuhr, J. and Schmitz, M.L., 2017. The NF-kB-dependent andindependent transcriptome and chromatin landscapes of human coronavirus 229Einfected cells. PLoS pathogens, 13(3), p.e1006286.

6. Andersen, K.G., Rambaut, A., Lipkin, W.I., Holmes, E.C. and Garry, R.F., 2020. The proximal origin of SARS-CoV-2. Nature medicine, 26(4), pp.450-452.

7. World Health Organization. Coronavirus disease (COVID-19) pandemic. https:// www.who.int/emergencies/diseases/novel-coronavirus-2019.

8. Jenner, R.G. and Young, R.A., 2005. Insights into host responses against pathogens from transcriptional profiling. Nature Reviews Microbiology, 3(4), pp.281-294.

9. Lin, L., Spoor, M.S., Gerth, A.J., Brody, S.L. and Peng, S.L., 2004. Modulation of Th1 activation and inflammation by the NF-kB repressor Foxj1. Science, 303(5660), pp. 1017-1020. 
10.Srivatsan, S. and Peng, S.L., 2005. Cutting edge: Foxj1 protects against autoimmunity and inhibits thymocyte egress. The Journal of Immunology, 175(12), pp.7805-7809.

11.Look, D.C., Walter, M.J., Williamson, M.R., Pang, L., You, Y., Sreshta, J.N., Johnson, J.E., Zander, D.S. and Brody, S.L., 2001. Effects of paramyxoviral infection on airway epithelial cell Foxj1 expression, ciliogenesis, and mucociliary function. The American journal of pathology, 159(6), pp.2055-2069.

12.Roy, S., Guler, R., Parihar, S.P., Schmeier, S., Kaczkowski, B., Nishimura, H., Shin, J.W., Negishi, Y., Ozturk, M., Hurdayal, R. and Kubosaki, A., 2015. Batf2/Irf1 induces inflammatory responses in classically activated macrophages, lipopolysaccharides, and mycobacterial infection. The Journal of Immunology, 194(12), pp.6035-6044.

13.Kitada, S., Kayama, H., Okuzaki, D., Koga, R., Kobayashi, M., Arima, Y., Kumanogoh, A., Murakami, M., Ikawa, M. and Takeda, K., 2017. BATF2 inhibits immunopathological Th17 responses by suppressing II23a expression during Trypanosoma cruzi infection. Journal of Experimental Medicine, 214(5), pp. 1313-1331.

PAGE 14 OF 23 
2

\begin{tabular}{|c|c|c|c|c|c|c|}
\hline Rank & ID & p-value & $\mathbf{F}$ & FC & Gene & Gene name \\
\hline 102 & A_23_P370682 & 1.49E-12 & 162.2162 & 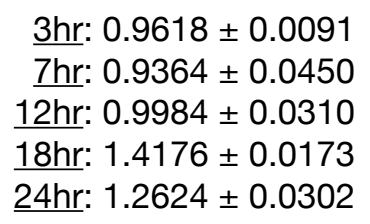 & BATF2 & $\begin{array}{l}\text { basic leucine } \\
\text { zipper transcription } \\
\text { factor, ATF-like } 2\end{array}$ \\
\hline 194 & A_23_P348636 & $9.27 \mathrm{E}-12$ & 126.57 & 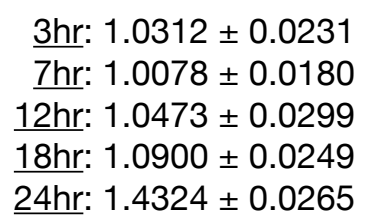 & FOXJ1 & forkhead box J1 \\
\hline
\end{tabular}

Table 1: BATF2 and FOXJ1 are differentially expressed in a human cell line following infection with an isolate of MERS-CoV, MERS-CoV London (Eng1).

The rank of differential expression, the probe ID, p-value of global differential expression, $\mathrm{F}$ statistic, fold change in BATF2 and FOXJ1 expression from 0-24 hours in MERS-CoV London-infected CALU3 cells, gene and gene name are listed in this chart. 


\begin{tabular}{|r|r|l|l|l|l|l|l|}
\hline Rank & ID & p-value & t & B & FC & Gene & Gene name \\
\hline 118 & 1336 & $3.06 \mathrm{E}-08$ & $2.35 \mathrm{E}+01$ & 9.84745 & $\begin{array}{l}1.3777 \pm \\
0.0299\end{array}$ & Batf2 & $\begin{array}{l}\text { basic leucine zipper } \\
\text { transcription factor, } \\
\text { ATF-like 2 }\end{array}$ \\
\hline $\mathbf{1 4 3 1 5}$ & 35566 & $4.92 \mathrm{E}-02$ & 2.35 & -5.14742 & & Foxj1 & forkhead box J1 \\
\hline
\end{tabular}

Table 2: Infection of BALB/c mice with SARS-CoV-1 results in differential expression of BATF2, and to a lesser extent, FOXJ1 in the lungs.

The rank of differential expression, the probe ID, the $p$-value of global differential expression, $t$, a moderated t-statistic, $B$, the log-odds of differential expression between the two groups compared (lungs of mock-infected mice vs. lungs of SARS-CoV-1infected mice), fold change in BATF2 expression in the lungs of BALB/c mice infected with SARS-CoV-1 as compared to mock-infected mice, gene and gene name are listed in this chart. 


\section{Table 3: Infection of C57BL6/NJ mice with SARS-CoV-1 results in differential} expression of BATF2, and to a lesser extent, FOXJ1 in the lungs.

The rank of differential expression, the probe ID, the $p$-value of global differential expression, $t$, a moderated t-statistic, $B$, the log-odds of differential expression between the two groups compared (lungs of mock-infected mice vs. lungs of SARS-CoV-1infected mice), gene and gene name are listed in this chart. 


\begin{tabular}{|l|l|l|l|l|l|}
\hline Rank & ID & p-value & F & Gene & Gene name \\
\hline $\mathbf{1 3 3 5 3}$ & CfaAffx.21637.1.S1_at & $1.41 \mathrm{E}-02$ & 7.96 & BATF2 & $\begin{array}{l}\text { basic leucine zipper } \\
\text { transcription factor, ATF-like 2 }\end{array}$ \\
\hline $\mathbf{2 7 3 4 0}$ & CfaAffx.8508.1.S1_at & $1.7 \mathrm{E}-01$ & 2.26 & FOXJ1 & forkhead box J1 \\
\hline
\end{tabular}

Table 4: Infection of ferrets with SARS-CoV-1 does not result in differential expression of FOXJ1 but does result in statistically significant differential expression of BATF2 in the lungs.

The rank of differential expression globally, the probe ID, the p-value of global differential expression, $\mathrm{F}$ statistic, gene and gene name are listed in this chart. 
Table 5: BATF2 and FOXJ1 are not differentially expressed in the blood of ferrets following intranasal SARS-CoV-1 infection.

The rank of differential expression globally, the probe ID, the p-value of global differential expression, $\mathrm{t}$, a moderated $\mathrm{t}$-statistic, $\mathrm{B}$, the log-odds of differential expression between the two groups compared (blood of mock-infected ferrets vs. blood of SARS-CoV-1-infected ferrets), gene and gene name are listed in this chart. 


\begin{tabular}{|c|l|l|l|l|l|l|}
\hline Rank & ID & p-value & t & B & Gene & Gene name \\
\hline $\mathbf{8 4 0 5}$ & 38840 & 0.0991907 & 1.9280332 & -4.1 & BATF2 & $\begin{array}{l}\text { basic leucine zipper } \\
\text { transcription factor, ATF-like 2 }\end{array}$ \\
\hline $\mathbf{2 9 2 2 1}$ & 24095 & 0.398083 & 0.9054783 & -4.991 & FOXJ1 & forkhead box J1 \\
\hline
\end{tabular}

Table 6: Infection of a human cell line with HCoV-229E does not result in differential expression of FOXJ1 and BATF2.

The rank of differential expression globally, the probe ID, the $p$-value of global differential expression, $t$, a moderated t-statistic, $B$, the log-odds of differential expression between the two groups compared (infected and uninfected cells), gene and gene name are listed in this chart. 


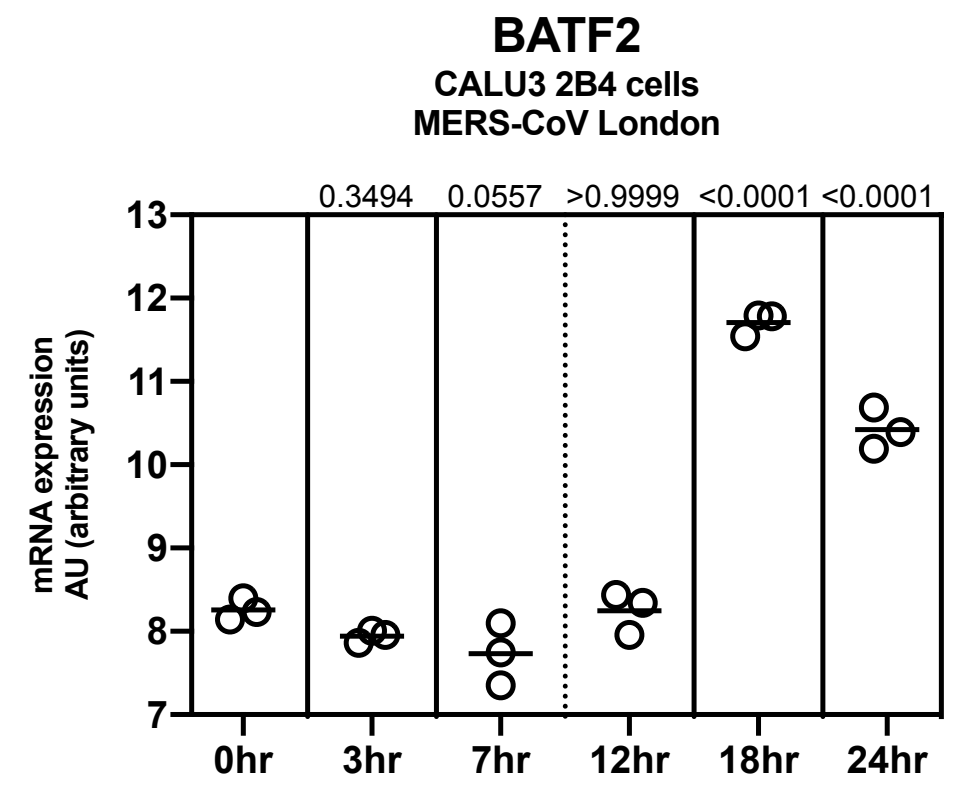

Figure 1: Infection of a human cell line with an isolate of MERS-CoV results in rapid transcriptional induction of BATF2.

BATF2 mRNA expression levels in CALU3 cells from 0-24 hours post-infection with MERS-CoV London (Eng1) are graphically represented here with the result of a statistical test evaluating the significance of difference in BATF2 expression as compared to baseline ( 0 hours), a $p$-value, listed above each time point. 


\section{FOXJ1}

CALU3 2B4 cells MERS-CoV London

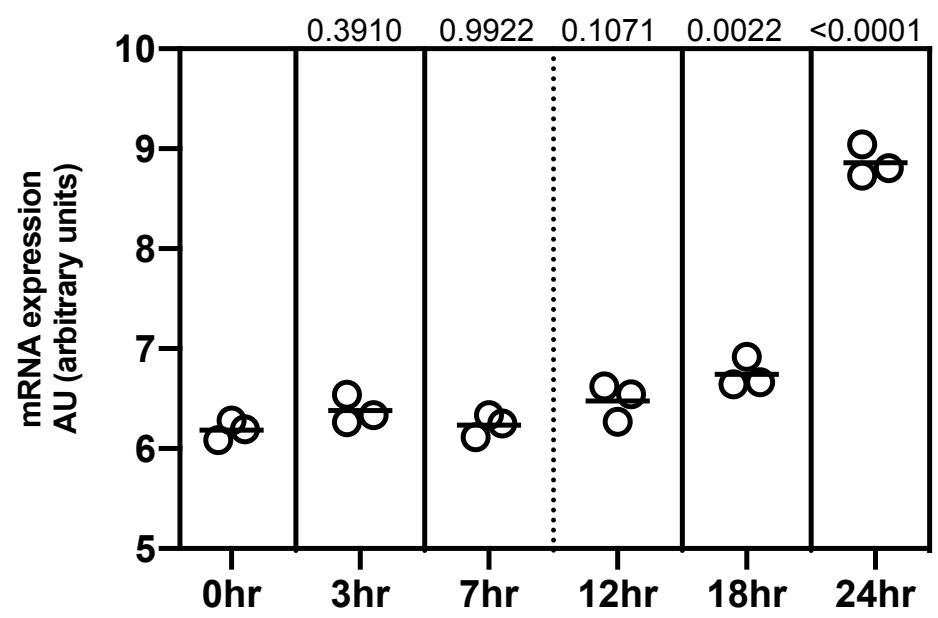

Figure 2: Infection of a human cell line with an isolate of MERS-CoV results in rapid transcriptional induction of FOXJ1.

FOXJ1 mRNA expression levels in CALU3 cells from 0-24 hours post-infection with MERS-CoV London (Eng1) are graphically represented here with the result of a statistical test evaluating the significance of difference in FOXJ1 expression as compared to baseline ( 0 hours), a $p$-value, listed above each time point. 


\section{BATF2}

Lungs of BALB/C mice infected with SARS-CoV

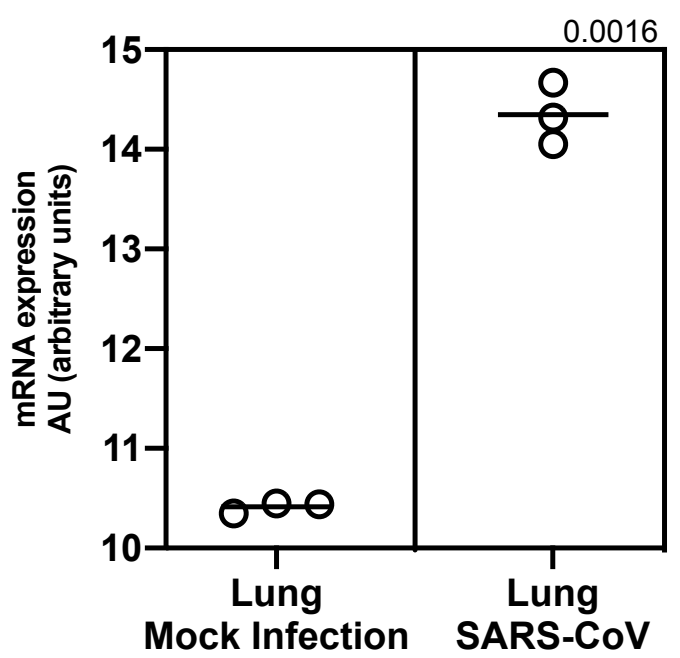

Figure 3: Infection of BALB/c mice with SARS-CoV-1 results in transcriptional induction of BATF2 in the lungs.

BATF2 mRNA levels are graphically represented here in the lungs of mock-infected mice (left) and in the lungs of SARS-CoV-1-infected mice at 2 days post-infection (right). The result of a statistical test evaluating significance of difference in BATF2 expression in the mouse lung at 2 days post-infection as compared to the mock-infected mouse lung, a $p$-value, is listed above. 